\title{
Epidemiologia de lesões musculoesqueléticas em praticantes amadores de futebol
}

\section{Epidemiology of musculoskeletal injuries in amateur soccer practitioners}

\author{
Marcus Victor Prudêncio Gonçalves ${ }^{1}$, Giuliano Moreto Onaka ${ }^{1}$, Dayana das Graças ${ }^{1}$, Rodrigo \\ Luiz Carregaro ${ }^{2}$, Paula Felippe Martinez ${ }^{1}$, Silvio Assis de Oliveira-Júnior ${ }^{1 *}$
}

\begin{abstract}
RESUMO
Este estudo teve por objetivo analisar a epidemiologia de lesões musculoesqueléticas (LME) no futebol, relacionando-as com frequência de prática e nível de atividade física. A casuística integrou 126 participantes homens, praticantes regulares de futebol amador em diferentes centros poliesportivos de Campo Grande/MS. Os participantes foram distribuídos em quatro grupos, de acordo com a frequência semanal de prática de futebol: G1, um dia, G2, dois dias, G3, três dias, e G4, quatro ou mais dias de atividade, com duração de 10 a 60 minutos. Para a tomada de informações sobre o nível de atividade física e LME, foram utilizados, respectivamente, o questionário internacional de atividade física (IPAQ) e um inquérito de morbidade referida. Não se constatou uma associação entre prática de futebol e nível de atividade física; o G4 mostrou maiores valores de gasto metabólico. Foram registrados 96 LME, envolvendo 87 participantes (69\%), totalizando 1,52 LME/ praticante. Constatou-se um predomínio de lesões musculares (36\%) e articulares (34\%) em membros inferiores. O trauma constituiu o principal mecanismo de lesão (28\%), destacando-se como a maior causa no G3. Foi possível constatar que a frequência de prática de futebol não se associa diretamente com a ocorrência de LME entre jogadores amadores de futebol.

Palavras-chave: futebol, atividade motora, traumatismos em atletas, fisioterapia
\end{abstract}

\begin{abstract}
The present study analyzed the athletic injuries prevalence among soccer practitioners according with the practice frequency and physical activity level. Studied sample included 126 regular and amateur soccer players in different sport centers from Campo Grande, MS, Brazil. The volunteers were distributed in four groups, according with week frequency of soccer practice: G1, one day; G2, two days; G3, three days; G4, four or more days of soccer practice for 10 until 60 minutes per day. Physical activity level was investigated with the International Physical Activity Questionnaire (IPAQ) while athletic injuries were studied with Health Survey. Soccer practice was not associated with physical activity level; G4 showed higher values of metabolic spending. 96 athletic injuries were verified in 87 soccer players (69\%), with occurrence of 1.52 injury/ practitioner. Muscle (36\%) and articular (34\%) damages were the more prevalent injury types; contact constituted the major etiologic mechanism to injury installation. As conclusion, the weekly practice magnitude had not associated with sports injuries occurrence in amateur soccer players.

Keywords: soccer, motor activity, athletic injuries, physical therapy
\end{abstract}

\footnotetext{
Artigo recebido a 14.11.2014; Aceite a 23.04.2015

${ }^{1}$ Universidade Federal de Mato Grosso do Sul, Campo Grande, MS, Brasil

${ }^{2}$ Universidade de Brasília (UnB), Ceilândia, DF, Brasil

* Autor correspondente: Cidade Universitária, S/n - Universitário, Campo Grande - MS, 79070-900, Brasil,

E-mail: oliveirajr.ufms@gmail.com
} 


\section{INTRODUÇÃO}

A associação entre a prática regular de exercícios físicos e os benefícios para a saúde humana está bem documentada na literatura (Reiner, Niermann, Jekauc, \& Woll, 2013). O exercício físico é uma opção de lazer e de restaurar a saúde dos efeitos nocivos decorrentes do sedentarismo. Após o período inicial de adaptação, a prática de exercício físico pode se tornar um hábito usualmente agradável, integrando inúmeros efeitos benéficos, inclusive, para a autoestima (Tofler, Mittleman, \& Muller, 1996). Segundo a American Heart Association (AHA) e o American College of Sports Medicine (ACSM), para promover e manter a saúde, adultos sadios precisam realizar exercício físico aeróbio com intensidade moderada por um período mínimo de 30 minutos por dia em cinco dias na semana, ou de intensidade vigorosa por um período mínimo de 20 minutos por dia em três dias semanais (Haskell et al., 2007). Combinações de exercícios físicos moderados e vigorosos podem ser realizadas para atender tais diretrizes (Cid, Silva, \& Alves, 2007).

Nesse contexto, a prática de futebol, seja por lazer ou competição, tem importância paradoxal. Embora configure a modalidade desportiva mais popular do mundo, com adeptos de várias nacionalidades, classes sociais e faixas etárias, o futebol é o desporto competitivo com maior incidência de lesões musculoesqueléticas (LME) (Bayraktar, Dinc, Yucesir, \& Evin, 2011; Ekstrand, Waldén, \& Hägglund, 2004; Fuller et al., 2006; Ribeiro, Vilaça, Oliveira, Vieira, \& Silva, 2007; Silveira et al., 2013). De fato, a prática futebolística é caracterizada pela ocorrência de contatos e solicitações físicomotoras específicas, incluindo-se corridas, saltos, arrancadas, mudanças de direção e quedas, que culminam em diferentes exigências de resistência, velocidade, agilidade, flexibilidade e força (Hoff, 2005; Manning \& Levy, 2006; Waldén, Hägglund, \& Ekstrand, 2005). Quando associadas ao contexto de uma competição, essas diversas condições podem contribuir para o desgaste físico e a fisiopatologia de LME, com a ressalva de que a sua etiologia é multifatorial, atrelada à interação entre diferentes fatores intrínsecos e/ou extrínsecos (Bahr \& Holme, 2003).

Sendo assim, o conhecimento da causa situacional, mecanismos de LME e fatores de risco relacionados ao nível de atividade física, pode auxiliar profissionais da área no processo de prevenção, diagnóstico e tratamento comuns ao futebol, a modalidade mais praticada no mundo (Fuller et al., 2006). Não obstante, embora diferentes estudos tenham se voltado a detalhar o perfil epidemiológico de LME entre futebolistas (Ribeiro et al., 2007; Santos, Assunção, Martinez, Christofoletti, \& Oliveira-Junior, 2014; Silveira et al., 2013), são escassas as evidências sobre a epidemiologia de LME entre praticantes amadores. Além disso, outra grande dificuldade reside na ausência de um instrumento clínico já estabelecido e validado para a avaliação e catalogação de lesões relacionadas a este públicoalvo.

Portanto, o presente trabalho teve por objetivo geral analisar a prevalência de LME em praticantes amadores de futebol, caracterizandoas segundo o nível de atividade física. O objetivo secundário foi descrever as lesões registradas, caracterizando-as segundo natureza, mecanismo de manifestação e local de acometimento, dentre outras informações epidemiológicas. Para tanto, utilizou-se de um inquérito de morbidade já empregado em estudos prévios com atletas profissionais (Santos et al., 2014; Silveira et al., 2013). Tem-se como hipótese inicial da pesquisa, que a incidência de LME é maior nos grupos com maior prática desportiva semanal e os agravos musculares em membros inferiores são as principais ocorrências notificadas.

\section{MÉTODO}

Trata-se de um estudo com delineamento transversal, de natureza observacional. $\mathrm{O}$ delineamento constituiu-se pela análise epidemiológica de diferentes grupos, definidos segundo a frequência semanal de prática de futebol, no contexto desportivo ou de lazer/ recreação. 


\section{Amostra}

Participaram da pesquisa 126 voluntários do sexo masculino, praticantes regulares de futebol amador em diferentes centros desportivos de Campo Grande/MS. Os participantes foram selecionados à sorte, de forma intencional adotando-se, como critério de inclusão, tempo mínimo de dois anos de prática regular e frequência de, pelo menos, uma vez por semana, com duração de 10 a 60 minutos. Os participantes foram informados sobre os objetivos da pesquisa, bem como do caráter voluntário de participação. Todos os sujeitos ou seus responsáveis assinaram o Termo de Consentimento Livre e Esclarecido. Esta pesquisa foi aprovada pelo Comitê de Ética em Pesquisa da UFMS, sob parecer 355.622/ 2013.

Os voluntários foram distribuídos em quatro grupos, definidos segundo a frequência semanal de prática desportiva ou de recreação. Sendo assim, o grupo I ( $\mathrm{GI} ; \mathrm{n}=36$ ) integrou praticantes com frequência de uma vez por semana. Os grupos II (G2; $\mathrm{n}=36)$, III (G3; $\mathrm{n}=28)$ e IV (G4; $\mathrm{n}=26$ ) foram constituídos por participantes que reportaram praticar duas, três e quatro ou mais ocasiões de jogo na semana, respetivamente.

\section{Instrumentos}

A aferição de estatura foi realizada com uso de uma fita métrica ajustada à parede e a massa corporal foi obtida com auxílio de uma balança digital (Leite et al., 2011; Sena et al., 2013).

Para a coleta de dados sobre LME, foram consideradas informações obtidas por meio do Inquérito de Morbidade Referida (IMR) (Pastre, Carvalho Filho, Monteiro, Netto Júnior, \& Padovani, 2004; Silveira et al., 2013). Este instrumento tem se mostrado útil para estudos epidemiológicos, sobretudo, pela objetividade e facilidade em se caracterizar o estado de agravos à saúde e suas características, como natureza, local de instalação, mecanismos de manifestação e necessidade de intervenção. Além disso, o inquérito conta com questões pertinentes sobre idade, massa corporal, estatura, histórico de prática, dominância de membros e motivação para a prática desportiva. Os dados foram coletados mediante abordagem e entrevista para registro de informações relacionadas às eventuais
LME desportivas correntes ao longo dos últimos 24 meses. Vale ressaltar que o processo foi realizado por um único pesquisador, treinado no manejo do IMR, desde a abordagem ao participante até as anotações protocolares e de procedimentos clínicos.

Para o detalhamento do nível de atividade física foi utilizado o Questionário Internacional de Atividade Física (IPAQ) (Matsudo et al., 2001). A forma longa do questionário incluindo questões sobre atividade física no trabalho, atividade física na locomoção, em casa (trabalho, tarefas domésticas e cuidar da família), na recreação, esporte, exercício e lazer, além de informações sobre tempo gasto sentado, foram utilizadas nas entrevistas, tomando como referência a última semana e explorando-se a periodicidade e duração da realização de cada atividade.

\section{Procedimentos}

Para caracterização dos grupos, além da idade, foram consideradas informações antropométricas, tempo de prática de futebol e epidemiologia de LME.

Como norteamento do presente estudo, um episódio de lesão foi definido como qualquer manifestação sintomática de dor ou disfunção musculoesquelética, decorrente da prática de futebol, e que tenha resultado em alterações na prática de jogo, seja na forma, duração, intensidade ou frequência de participações (Bennell \& Crossley, 1996).

O nível de atividade física foi classificado em três categorias (Brito et al., 2012; Matsudo et al., 2001):

1. Baixo: não atingiu níveis de atividade física adequados para serem incluídos nas categorias dois e três (a seguir);

2. Moderado:

2.1. Atividade física intensa: 3 dias/semana e $20 \mathrm{~min} /$ dia; ou

2.2. Moderada ou caminhada: 5 dias/semana e $30 \mathrm{~min} / \mathrm{dia}$; ou

2.3. Qualquer atividade somada: 5 dias/semana de qualquer combinação entre caminhada e exercício moderado ou intenso. Atingir ao menos o total de 
600 equivalentes metabólicos (MET) min/semana;

3. Alto:

3.1. Atividade física intensa: 3 dias/semana. Atingir ao menos o total de $1.500 \mathrm{MET} / \mathrm{min} / \mathrm{semana}$; ou

3.2. Qualquer atividade somada: sete dias/semana de qualquer combinação entre caminhada e exercício moderado ou intenso, atingindo ao menos 3.000 $\mathrm{MET} / \mathrm{min} / \mathrm{semana}$.

\section{Análise estatística}

Para a análise dos resultados relativos ao perfil demográfico e antropométrico, assim como histórico de prática e nível de atividade física, foi utilizada análise de variância (ANOVA), complementada com teste de comparações múltiplas de Student-Newman-Keuls.

Para a análise dos resultados descritivos de LME, considerou-se o teste de proporções multinomiais de Goodman (Goodman, 1965). A significância adotada foi de $5 \%$.

\section{RESULTADOS}

As informações sobre caracterização dos grupos, considerando-se perfil demográfico e antropométrico, histórico de prática desportiva e gasto energético, são apresentadas na Tabela 1 . Não foram constatadas diferenças significantes relativas aos dados demográficos e antropométricos, entre os grupos sob estudo. No entanto, o histórico de prática mostrou-se significativamente maior no G3, quando comparado ao G1, e o G4 apresentou maiores valores de gasto energético em atividades de moderada intensidade e atividade física total em relação aos demais grupos (Tabela 1).

No contexto da classificação segundo os níveis de atividade física, enquanto a maioria dos constituintes de G1 enquadrou-se nas classes de baixa e alta exigência física, a maior parte dos participantes do G4 (62\%) integrou a classe de alto nível de atividade física. Nos grupos G2 e G3, a maior parcela de voluntários situou-se no nível de moderada demanda físico-motora (Tabela 2).

Tabela 1

Perfil demográfico e antropométrico, histórico de prática (HP) e gasto energético (METs) em diferentes condições de atividade física de acordo com o grupo

\begin{tabular}{lcccc}
\hline Variáveis & \multicolumn{3}{c}{ Grupo } & G4 \\
\cline { 2 - 5 } & G1 & G2 & G3 & $81 \pm 15$ \\
Massa $(\mathrm{kg})$ & $77 \pm 9$ & $81 \pm 13$ & $176 \pm 8$ & $177 \pm 7$ \\
Estatura (cm) & $177 \pm 6$ & $180 \pm 7$ & $29 \pm 12$ & $25 \pm 10$ \\
Idade (anos) & $25 \pm 9$ & $25 \pm 10$ & $15.0 \pm 17.0 *$ & $8.0 \pm 9.3$ \\
HP (anos) & $12.0 \pm 6.0$ & $12.5 \pm 6.5$ & $363 \pm 1931$ & $495 \pm 990$ \\
METs caminhada & $256 \pm 594$ & $396 \pm 594$ & $1170 \pm 1215$ & $2760 \pm 2160^{* \# \$}$ \\
METs moderada & $685 \pm 1370$ & $1320 \pm 1035$ & $1850 \pm 750$ & $1348 \pm 395$ \\
METs vigorosa & $662 \pm 217$ & $1116 \pm 509$ & $2091 \pm 4602$ & $3946 \pm 4251^{* *}$ \\
METs total & $1398 \pm 3990$ & $1940 \pm 2627$ & $3084 \pm 304$ & $2766 \pm 258$ \\
METs sentado & $3514 \pm 283$ & $3692 \pm 272$ &
\end{tabular}

Resultados de massa, estatura, idade e gasto energético expressos em média \pm desvio-padrão; resultados de HP mostrados em mediana \pm intervalo interquartílico; * $\mathrm{p}<0.05$ versus $\mathrm{G} 1 ;{ }^{*} \mathrm{p}<0.05$ versus $\mathrm{G} 2{ }^{\$}{ }^{\$} \mathrm{p}<0.05$ versus $\mathrm{G} 3$

Tabela 2

Distribuição absoluta e relativa dos participantes segundo nível de atividade física

\begin{tabular}{lcccc}
\hline Nível de atividade física & \multicolumn{4}{c}{ Grupo } \\
\cline { 2 - 5 } & $\mathrm{G} 1$ & $\mathrm{G} 2$ & $\mathrm{G} 3$ & $\mathrm{G} 4$ \\
\hline Baixo & $14(38.9 \%)^{\mathrm{Ab}}$ & $11(30.6 \%)^{\mathrm{Ab}}$ & $4(14.3 \%)^{\mathrm{Aab}}$ & $1(3.8 \%)^{\mathrm{Aa}}$ \\
Moderado & $7(19.4 \%)^{\mathrm{Aa}}$ & $14(38.9 \%)^{\mathrm{Aa}}$ & $14(50.0 \%)^{\mathrm{Aa}}$ & $9(34.6 \%)^{\mathrm{Ba}}$ \\
Alto & $15(41.7 \%)^{\mathrm{Aa}}$ & $11(30.6 \%)^{\mathrm{Aa}}$ & $10(35.7 \%)^{\mathrm{Aa}}$ & $16(61.6 \%)^{\mathrm{Ba}}$
\end{tabular}

Resultados expressos em frequência absoluta e relativa (entre parênteses); ${ }^{\mathrm{A}, \mathrm{B}}$ : comparações verticais, segundo nível de atividade física, sendo $\mathrm{A}<\mathrm{B}$; ${ }^{\mathrm{a}, \mathrm{b}}$ : comparações horizontais, segundo grupo, sendo $\mathrm{a}<\mathrm{b}$. Teste de Goodman para contrastes entre e dentro de populações multinomiais 
No contexto epidemiológico, a prevalência de praticantes com registro de LME mostrou-se maior no $\mathrm{G} 3(\mathrm{n}=18 ; 64 \%)$, acompanhado por $\mathrm{G} 1$ $(\mathrm{n}=20 ; 56 \%), \mathrm{G} 4(\mathrm{n}=14 ; 54 \%)$ e $\mathrm{G} 2(\mathrm{n}=16$; $44 \%)$. Similarmente, a taxa de lesão foi maior no G3 (0.82 LME/indivíduo), seguido por G2 (0.77 LME/indivíduo), G1 (0.75 LME/ indivíduo) e G4 (0.69 LME/indivíduo). Considerando-se a taxa de lesão por indivíduo, têm-se as taxas de 1,8 casos/ indivíduo no G1, 1.75 casos/ indivíduo no G2, 1.27 casos/ indivíduo no G3 e 1.28 casos/indivíduo no G4.

Levando-se em conta a natureza das afeções, as lesões musculares foram as mais relatadas pelos atletas (36\%), seguidas por lesões articulares $(34 \%) \quad(\mathrm{p}<0.05)$. No contexto descritivo, G4 apresentou o maior número de casos de lesões musculares (11 LME; 69\%); já as lesões articulares mostraram-se mais prevalentes no G2 (11 LME; 69\%) (Figura 1).

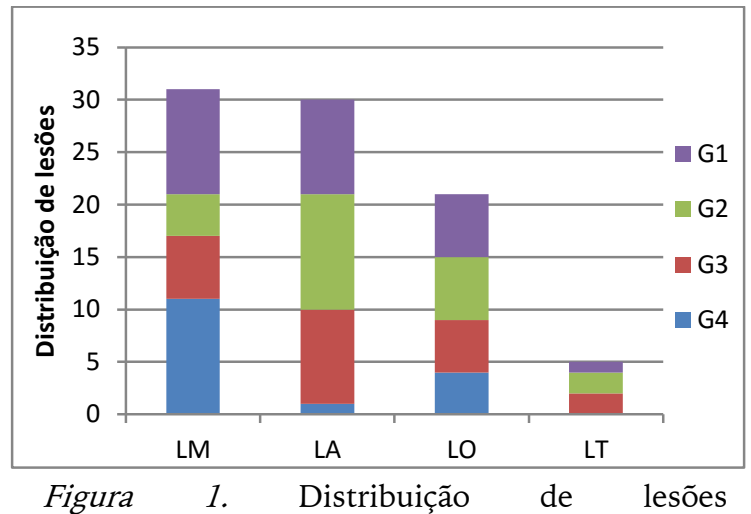
musculoesqueléticas, segundo grupo de estudo e natureza; LM: lesão muscular; LA: lesão articular; LO: lesão óssea; LT: lesão tendínea

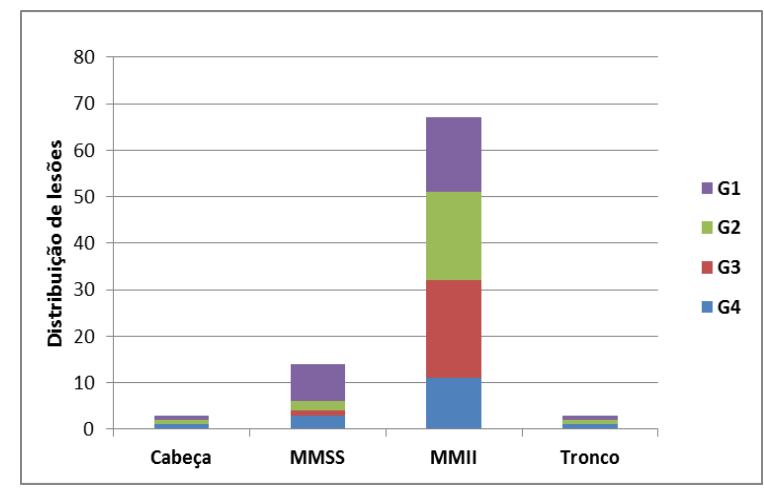

Figura 2. Distribuição absoluta e relativa (\%) de lesões musculoesqueléticas segundo grupo e segmento anatômico de instalação; MMSS: membros superiores; MMII: membros inferiores
Quanto à localização anatômica dos acometimentos, houve uma grande prevalência de lesões em extremidades inferiores (67 LME; $77 \%$; $\mathrm{p}<0.05)$, em relação às demais regiões anatômicas (Figura 2). Por sua vez, dentre os mecanismos de causa de LME, o trauma foi o principal fator etiológico das notificações de lesão (28\%; p <0.05). Esse achado foi também observado em todos os grupos (Figura 3 ).

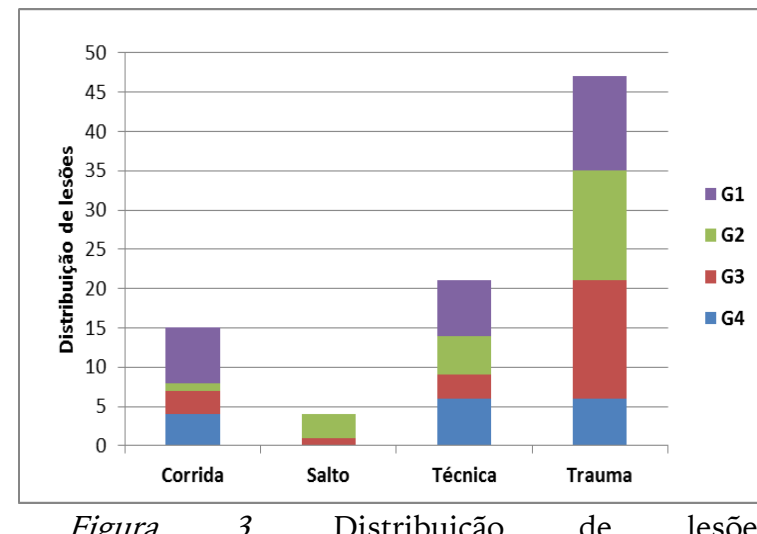
musculoesqueléticas segundo grupo e mecanismo de manifestação

Em relação à requisição por tratamento, a maioria das ocorrências culminou em procura por assistência terapêutica $\quad(n=57 ; 66 \%)$; situação semelhante foi observada em todos os grupos estudados. Já nos retornos, quanto à manifestação de sintomas, 15 (33\%), 13 (29\%), $7(16 \%)$ e 10 (22\%) participantes pertenciam, respetivamente, aos grupos G1, G2, G3 e G4. Entretanto, essa distribuição não foi diferente entre os grupos ( $p>0.05)$.

\section{DISCUSSÃO}

O presente estudo foi realizado com o propósito de analisar a epidemiologia das lesões musculoesqueléticas em praticantes amadores de futebol, relacionando-as com a frequência semanal de prática. Em contraste à hipótese inicial da investigação, a incidência de LME mostrou-se maior entre praticantes regulares de futebol em frequência de duas a três vezes por semana, classificados como sujeitos de moderado nível de atividade física. As lesões musculares em membros inferiores confirmaram-se como as principais ocorrências registradas; no entanto, tal 
achado não se associou diretamente com a frequência de prática desportiva e da demanda físico-motora.

No aspeto epidemiológico, levando-se em conta as maiores taxas de prevalência de LME em G1 e G3 e as menores prevalências de afecções em G4, pode-se inferir que a maior demanda por atividades de intensidade moderada e vigorosa, incluindo-se a prática semanal de futebol, pode ter repercutido em maior adaptação e melhor aptidão físico-motora nos participantes do G4, como efeitos comuns do exercício físico regular (McArdle, Katch, \& Katch, 2010). Em relação às afecções, a maioria dos registros integrou lesões musculares, o que também se confirmou apenas no G4. Os resultados podem estar ligados a repetitivas exigências de jogo, envolvendo gestual específico do futebol, como chute, explosão e corrida (Hoff, 2005; Manning \& Levy, 2006), executados em maior frequência. Neste sentido, a maior exposição à sobrecarga do exercício pode ter repercutido em uma maior demanda musculoesquelética, devido aos gestos desportivos e natureza competitiva da prática de futebol (Hoff, 2005). Outras desordens, contudo, como alterações posturais e desequilíbrios musculares (Sena et al., 2013), também poderiam contribuir para a ocorrência de LME no G4, atuando como fatores de risco intrínseco a esta população.

As notificações dos grupos G1 e G3 revelaram proporções similares entre lesões musculares e articulares, enquanto no G2, a maior parte dos achados envolveu lesões articulares. As altas taxas de afecções articulares nesses grupos podem estar associadas com menor capacidade proprioceptiva e estabilidade articular dinâmica (Leporace, Metsavaht, \& Sposito, 2009). Esses atributos são determinados pela interação entre diferentes fatores relacionados com a sensação mecânica ligamentar e articular, decorrente de forças compressivas, gravidade e atividade muscular, em intensidade e frequência de ocorrências. Considerando-se que as atividades moderadas e vigorosas foram similares entre G1, G2 e G3, e menores do que em G4, é possível que as adaptações sensório-motoras e musculares provindas do exercício não tenham se consolidado nesses grupos. Além disso, não se pode descartar a maior proporção de recidivas, especialmente nos grupos G1 e G2, que reportaram taxas de 1.8 e 1.75 casos/indivíduo, respetivamente. Alguns estudos demonstraram diminuição da propriocepção e da resposta motora, em decorrência de lesões musculoesqueléticas (Myers et al., 2004; Swanick, Lephart, Giannantonio, \& Fu, 1997), o que corrobora nossos achados. De fato, os grupos G1 e G2 exibiram as maiores taxas de manifestação sintomática ao retorno da prática de futebol, o que pode subsidiar a maior proporção de afecções articulares nesses grupos. Silva, Abdalla, e Fisberg (2007) constataram que lesões de tecidos articulares e periarticulares demandam maior tempo de regeneração e retorno funcional, em relação a afecções musculares, entre jogadoras de basquetebol.

Quanto ao local das lesões, houve maior prevalência de acometimentos em membros inferiores, os quais são decorrentes, principalmente, de situações de contato, confirmando evidências prévias da literatura (Santos et al., 2014; Silveira et al., 2013). Este achado está associado também a exigências típicas do futebol, incluindo-se solicitações específicas de saltos, aterrissagens, mudanças de direção, chutes e pivoteios que, por si só, constituem importantes causas extrínsecas para a instalação de LME (Bahr \& Holme, 2003). Silveira et al. (2013) verificaram que a maioria das afecções musculoesqueléticas resultou em procura por acompanhamento terapêutico, evidenciando a gravidade de tais acometimentos, ainda que grande parte dos agravos tenha culminado em retorno assintomático, como observado no presente trabalho.

Embora o presente manuscrito abranja uma temática inovadora em relação ao público estudado, a relação entre frequência de prática desportiva e nível de atividade física na fisiopatologia de lesões musculoesqueléticas ainda carece de maior exploração. Estudos com delineamento longitudinal poderão melhor esclarecer as potenciais relações entre os assuntos abordados.

Ademais, outra importante limitação integra a imprecisão no desfecho clínico das ocorrências listadas no presente estudo. Embora a maioria 
dos casos tenha culminado em busca por assistência médico-terapêutica, o momento de alta e retorno funcional é, de certo modo, impreciso. A relação entre tratamento e sintomatologia remete à hipótese de que praticantes amadores de futebol possam apresentar lesões de maior gravidade e morbidade, em decorrência do menor condicionamento físico. Diante dos presentes achados, no entanto, pode-se pressupor que a busca por abordagem terapêutica foi suficiente para a resolução definitiva dos agravos registrados. Contudo, importantes fatores de risco intrínseco para a instalação de lesões, como alterações anatômicas e posturais (Sena et al., 2013), além de atributos extrínsecos, como o tipo de solo e vestimentas (Bahr \& Holme, 2003), não foram avaliados. Tais fatores poderiam contribuir para a ocorrência de novas lesões e levar a possíveis recidivas de agravos pré-existentes.

\section{CONCLUSÕES}

A frequência de prática de futebol não se associou com a incidência de lesões musculoesqueléticas entre jogadores amadores de futebol. As lesões musculares em membros inferiores e decorrentes de trauma foram os principais agravos clínicos registrados.

\section{Agradecimentos:}

Nada a declarar.

\section{Conflito de Interesses:}

Nada a declarar

\section{Financiamento:}

Nada a declarar

\section{REFERÊNCIAS}

Bahr, R., \& Holme, I. (2003). Risk factors for sports injuries - a methodological approach. British Journal of Sports Medicine, 37(5), 384-392. http://doi.org/10.1136/bjsm.37.5.384

Bayraktar, B., Dinc, C., Yucesir, I., \& Evin, A. (2011). Injury evaluation of the Turkish national football team over six consecutive seasons. Turkish Journal of Trauma and Emergency Surgery,
17(4), $313-317$. http://doi.org/10.5505/tjtes.2011.86836

Bennell, K. L., \& Crossley, K. (1996). Musculoskeletal injuries in track and field: incidence, distribution and risk factors. Australian Journal of Science and Medicine in Sport, 28(3), 69-75.

Brito, W. F., Santos, C. L. dos, Marcolongo, A. do A., Campos, M. D., Bocalini, D. S., Antonio, E. L., ... Serra, A. J. (2012). Nível de atividade física em professores da rede estadual de ensino. Revista de Saúde Pública, 46(1), 104-109. http://doi.org/10.1590/S003489102012000100013

Cid, L., Silva, C., \& Alves, J. (2007). Actividade física e bem-estar psicológico - perfil dos participantes no programa de exercício e saúde de rio maior. Motricidade, 3(2), 47-55. http://doi.org/10.6063/motricidade.3(2).674

Ekstrand, J., Waldén, M., \& Hägglund, M. (2004). Risk for injury when playing in a national football team. Scandinavian Journal of Medicine \& Science in Sports, 14(1), 34-38.

Fuller, C. W., Ekstrand, J., Junge, A., Andersen, T. E., Bahr, R., Dvorak, J., ... Meeuwisse, W. H. (2006). Consensus statement on injury definitions and data collection procedures in studies of football (soccer) injuries. Scandinavian Journal of Medicine \& Science in Sports, 16(2), 83-92. http://doi.org/10.1111/j.16000838.2006.00528.x

Goodman, L. A. (1965). On simultaneous confidence intervals for multinomial proportions. Technometrics, $\quad 7(2), \quad 247-254$. http://doi.org/10.2307/1266673

Haskell, W. L., Lee, I.-M., Pate, R. R., Powell, K. E., Blair, S. N., Franklin, B. A., ... Bauman, A. (2007). Physical activity and public health: updated recommendation for adults from the American College of Sports Medicine and the American Heart Association. Circulation, 116(9), 1081-1093. http://doi.org/10.1161/CIRCULATIONAHA.10 7.185649

Hoff, J. (2005). Training and testing physical capacities for elite soccer players. Journal of Sports Sciences, 23(6), 573-582. http://doi.org/10.1080/02640410400021252

Leite, N., Junior, A., De, R. P., Cieslak, F., Ishiyama, M., Milano, G. E., \& Stefanello, J. M. F. (2011). Perfil da aptidão física dos praticantes de Le Parkour. Revista Brasileira de Medicina do Esporte, 17(3), 198-201. http://doi.org/10.1590/S151786922011000300010

Leporace, G., Metsavaht, L., \& Sposito, M. M. de M. (2009). Importância do treinamento da propriocepção e do controle motor na reabilitação após lesões músculo-esqueléticas. Revista Acta Fisiátrica, 16(3), 126-131.

Manning, M. R., \& Levy, R. S. (2006). Soccer. Physical Medicine and Rehabilitation Clinics of North 
America, $\quad$ 17(3), 677-695. http://doi.org/10.1016/j.pmr.2006.05.004

Matsudo, S., Araújo, T., Matsudo, V., Andrade, D., Andrade, E., Oliveira, L. C., \& Braggion, G. (2001). Questionário internacional de atividade física (Ipaq): estudo de validade e reprodutibilidade no Brasil. Revista Brasileira de Atividade Física \& Saúde, 6(2), 5-18.

McArdle, W. D., Katch, F. I., \& Katch, V. L. (2010). Exercise Physiology: Nutrition, Energy, and Human Performance (3rd ed.). Philadelphia: Lippincott Williams \& Wilkins.

Myers, J. B., Ju, Y.-Y., Hwang, J.-H., McMahon, P. J., Rodosky, M. W., \& Lephart, S. M. (2004). Reflexive muscle activation alterations in shoulders with anterior glenohumeral instability. The American Journal of Sports Medicine, 32(4), 1013-1021.

Pastre, C. M., Carvalho Filho, G., Monteiro, H. L., Netto Júnior, J., \& Padovani, C. R. (2004). Sports injuries in track and field: comparison between information obtained in medical records and reported morbidity inquires. Revista Brasileira de Medicina do Esporte, 10(1), 1-8. http://doi.org/10.1590/S151786922004000100001

Reiner, M., Niermann, C., Jekauc, D., \& Woll, A. (2013). Long-term health benefits of physical activity - a systematic review of longitudinal studies. BMC Public Health, 13, 813. http://doi.org/10.1186/1471-2458-13-813

Ribeiro, R. N., Vilaça, F., Oliveira, H. U. de, Vieira, L. S., \& Silva, A. A. da. (2007). Prevalência de lesões no futebol em atletas jovens: estudo comparativo entre diferentes categorias. Revista Brasileira de Educação Física e Esporte, 21(3), 189-194. http://doi.org/10.1590/S180755092007000300003

Santos, G. de P., Assunção, V. H. S. de, Martinez, P. F., Christofoletti, G., \& Oliveira-Junior, S. A. (2014). Incidência de lesões desportivas e supratreinamento no futebol. ConScientiae Saúde, 13(2), 203-210. http://doi.org/10.5585/conssaude.v13n2.4735

Sena, D. A. de, Ferreira, F. M., Melo, R. H. G. de, Taciro, C., Carregaro, R. L., Júnior, O., \& De, S. A. (2013). Analysis of the joint flexibility and prevalence of soccer injuries according to age. Fisioterapia e Pesquisa, 20(4), 343-348. http://doi.org/10.1590/S180929502013000400007

Silva, A. S. da, Abdalla, R. J., \& Fisberg, M. (2007). Incidência de lesões musculoesqueléticas em atletas de elite do basquetebol feminino. Acta Ortopédica Brasileira, 15(1), 43-46. http://doi.org/10.1590/S141378522007000100009

Silveira, K. P., Assunção, V. H. S., Guimarães-Junior, N. P., Miziara-Barbosa, S. R., Santos, M. L. M., Christofoletti, G., ... Oliveira-Junior, S. A. (2013). Nosographic profile of soccer injuries according to the age group. Brazilian Journal of Kinanthropometry and Human Performance, 15(4), 476-485. http://doi.org/10.5007/19800037.2013v15n4p476

Swanick, C. B., Lephart, S. M., Giannantonio, F. P., \& Fu, F. H. (1997). Re-establishing proprioception and neuromuscular control in the ACL-injured athlete. Journal of Sport Rehabilitation, 6(2), 182-206.

Tofler, G. H., Mittleman, M. A., \& Muller, J. E. (1996). Physical activity and the triggering of myocardial infarction: the case for regular exercise. Heart, 75(4), 323-325.

Waldén, M., Hägglund, M., \& Ekstrand, J. (2005). Injuries in Swedish elite football-a prospective study on injury definitions, risk for injury and injury pattern during 2001. Scandinavian Journal of Medicine \& Science in Sports, 15(2), 118-125. http://doi.org/10.1111/j.16000838.2004.00393.x 